\title{
Estimating the leaf area of an individual tree in urban areas using terrestrial laser scanner and path length distribution model
}

\author{
Ronghai Hu, b, c, Elena Bournez ${ }^{\mathrm{b}}$, Shiyu Cheng ${ }^{\mathrm{a}, \mathrm{b}}$, Hailan Jianga, b, Françoise Nerry ${ }^{\mathrm{c}}$, Tania Landes ${ }^{\mathrm{c}}$, \\ Marc Saudreau $^{\mathrm{d}}$, Pierre Kastendeuch ${ }^{\mathrm{c}}$, Georges Najjarc, Jérôme Colin ${ }^{\mathrm{c}}$, Guangjian Yan ${ }^{\mathrm{a}, \mathrm{b}}$, \\ ${ }^{a}$ State Key Laboratory of Remote Sensing Science, Jointly Sponsored by Beijing Normal University and Institute of Remote Sensing and Digital Earth of Chinese Academy of Sciences, Beijing \\ 100875, China \\ ${ }^{\mathrm{b}}$ Beijing Engineering Research Center for Global Land Remote Sensing Products, Faculty of Geographical Science, Beijing Normal University, Beijing 100875, China \\ ${ }^{c}$ ICube Laboratory, UMR 7357 CNRS-University of Strasbourg, 300 bd Sebastien Brant, CS 10413, F-67412 Illkirch Cedex, France \\ ${ }^{\mathrm{d}}$ University of Clermont Auvergne, INRA, PIAF, F-63000 Clermont Ferrand, France
}

\section{A R T I C L E I N F O}

Keywords:

Individual tree

Leaf area

Foliage area volume density

Terrestrial laser scanner

Urban areas

Path length distribution

\begin{abstract}
A B S T R A C T
Urban leaf area measurement is crucial to properly determining the effect of urban trees on micro-climate regulation, heat island effect, building cooling, air quality improvement, and ozone formation. Previous works on the leaf area measurement have mainly focused on the stand level, although the presence of individual trees is more common than forests in urban areas. The only feasible ways for an operational non-destructive leaf area measurement, namely, optical indirect methods, are mostly limited in urban areas because light path is constantly intercepted by surrounding buildings or other objects. A terrestrial laser scanner (TLS), which can extract an individual tree by using its unique distance information, provides a possibility for indirectly measuring the leaf area index (LAI) in urban areas. However, indirect LAI measurement theory, which uses the cosine of an observation zenith angle for path-length correction, is incompatible for an individual tree because the representative projected area of LAI changes while the observation zenith angle changes, thus making the results incomparable and ambiguous. Therefore, we modified a path length distribution model for the leaf area measurement of an individual tree by replacing the traditional cosine path length correction for a continuous canopy with real path length distribution. We reconstructed the tree crown envelope from a TLS point cloud and calculated a real path length distribution through laser pulse-envelope intersections. Consequently, leaf area density was separated from the path length distribution model for leaf area calculation. Comparisons with reference measurement for an individual tree showed that the TLS-derived leaf area using the path length distribution is insensitive to the scanning resolution and agrees well with an allometric measurement with an overestimation from $5 \mathrm{~m}^{2}$ to $18 \mathrm{~m}^{2}(3-10 \%$, respectively). Results from different stations are globally consistent, and using a weighted mean for different stations by sample numbers further improves the universality and efficiency of the proposed method. Further automation of the proposed method can facilitate a rapid and operational leaf area extraction of an individual tree for urban climate modeling.
\end{abstract}

\section{Introduction}

Leaf area parameters, generally expressed as leaf area index (LAI) and leaf area density (LAD, also referred as foliage area volume density, FAVD), are important parameters for describing a canopy and crown structure given their close relation to many biological and physical processes, such as photosynthesis, respiration, transpiration, and carbon and nutrient cycling (Chen and Cihlar, 1996; Jupp et al., 2009; Ren et al., 2013; Tian et al., 2015). Urban leaf area has increasingly attracted attention considering its important role in micro-climate regulation, heat island effect mitigation, building cooling (reducing energy consumption), air quality improvement, and ozone formation (Alonzo et al., 2015; Benjamin and Winer, 1998; Najjar et al., 2015; Peper and McPherson, 1998; Simpson, 1998). Accurate leaf area measurement for urban trees is required to properly determine their benefits.

\footnotetext{
* Corresponding author at: State Key Laboratory of Remote Sensing Science, Jointly Sponsored by Beijing Normal University and Institute of Remote Sensing and Digital Earth of Chinese Academy of Sciences, Beijing 100875, China.

Email address: gjyan@bnu.edu.cn (G. Yan)
} 
Previous works on leaf area measurement have mainly focused on the stand level, that is, a contiguous community of trees (Leblanc and Fournier, 2014; Nowak et al., 2008; Strahler et al., 2008; Weiss et al., 2004). The leaf area measurement for an individual tree is rarely explored because isolated trees are more common than forests in urban areas. Methods for ground leaf area measurement can be classified as direct and indirect. Direct methods, such as harvesting, allometry, and litter collection, can be used for measuring an individual tree and are considered more accurate than the indirect methods (Colaizzi et al., 2017; Daughtry, 1990; Peper and McPherson, 2003; Simioni et al., 2004). However, these methods exert disadvantages, such as time-consuming, labor-intensive, and destructive to vegetation and thus are unable to complete long-term monitoring of spatial and temporal dynamics of leaf area development (Jonckheere et al., 2004). Indirect methods, in which the leaf area is inferred from measurements of other variables, such as gap probability or light transmission through canopies, are efficient, nondestructive, and amendable to automation; thus, these methods are extensively used (Macfarlane et al., 2014; Mu et al., 2017; Ryu et al., 2010; Yao et al., 2011). Most indirect methods rely on optical instruments, such as LAI-2000, TRAC, and HemiView (Chen, 1996; Leblanc et al., 2005). However, these instruments are inapplicable to urban individual tree LAI measurement because their light paths are constantly intercepted by surrounding buildings or other objects. Therefore, new instruments and methods to separate individual trees from the urban environment must be explored.

Light detection and ranging (LiDAR) is an active remote-sensing technology, which captures three-dimensional (3D) point clouds of the scanned object, thereby providing an opportunity to extract the 3D geometry of an individual tree. This technology has been extensively used in obtaining the parameters, such as tree height, diameter at breast height (DBH), canopy density, and biomass, of canopies and crowns (Bouvier et al., 2015; Koch et al., 2006; Wang et al., 2009). Moreover, a terrestrial laser scanner (TLS), which is used in measuring vegetation structure information, has advantages, such as favorable directivity, high angular resolution, and strong anti-interference capability. Another advantage of the TLS for individual tree measurement is its capability to separate the studied tree from the urban environment using its unique distance information. Three methods, namely, regression-, voxel-, and gap probability-based, have been implemented using the TLS to obtain the leaf area of an individual tree. The regression-based method is used to estimate an individual tree leaf area on the basis of a regression model with LiDAR-derived tree dimensions, such as tree height and crown diameter (Roberts et al., 2003). This method is less laborious and more efficient than the traditional allometric method because this method uses LiDAR-derived tree dimensions rather than manually measuring the tree dimensions for regression. However, the regression-based method still relies on manually measuring the leaf area of several trees to establish a regression relationship, and the accuracy of this method is largely limited by the accuracy of the LiDAR-based estimates of crown dimensions (Roberts et al., 2005). The voxel-based 3D modeling method is used to estimate the LAI and leaf area density by directly counting the contact frequency in each layer of the studied tree (Beland et al., 2014; Grau et al., 2017; Hosoi and Omasa, 2006). This method is non-destructive but inappropriate for measuring multiple trees given its requirement for fully and evenly scanned data from several stations for every tree. The gap probability-based method is used to calculate the LAI from the gap probability or gap size distribution based on Beer's law. This method is explored further than the other methods given its developed theory and operational and efficient measurement scheme for continuous canopies (Chianucci et al., 2015; Lin and West, 2016; Moorthy et al., 2008; Xie et al., 2017). However, most gap probability-based methods at the stand level require an adjustment in terms of the individual tree because the continuous canopy assumption is typically unsatisfied. Two theoretical problems in Beer's law for individual tree measurement are presented as follows: (1) the representative projected area changes with the change in an observation zenith angle (Nilson, 1999) and (2) a large proportion of laser pulses are distributed in large zenith angles near $90^{\circ}$ considering the relative position and height between the TLS station and the tree crown, which can hardly be used in the traditional methods (Demarez et al., 2008; Gonsamo and Pellikka, 2009). Moreover, the 3D LiDAR data are not fully explored in the gap probability-based method because the gap probability or gap size distribution used in these methods only has 2D information.

A path length distribution model (known as the PATH method) is a Beer's law-based method, which considers various path lengths within the crowns (Hu et al., 2014) and has a potential for using the 3D information of LiDAR, that is, distance (Hu et al., 2018). The path length distribution model is not limited by the observation zenith angle because this model replaces the traditional cosine path-length correction for a continuous canopy with a real path length distribution. This model is originally used to measure the LAI of the continuous canopy and derives the relative path length distribution from the gap probability distribution using a sliding window (Hu et al., 2016; Yan et al., 2016; Zeng et al., 2015). The unique distance information of the LiDAR provides a potential to obtain the accurate path length information directly for the path length distribution model, thereby possibly separating the path length and leaf area density. Moreover, the model can also be used to eliminate the influence of the laser pulses reflected by the objects rather than the target tree. Owing to these findings, the proposed method adjusted the path length distribution model to an individual tree to calculate the leaf area density and leaf area by building an envelope using the LiDAR data to obtain the absolute path length.

\section{Modeling the leaf area of a single tree}

\subsection{Beer's law for a forest stand}

The general formula of the Beer's law-based method is developed for a forest stand as follows:

$P(\theta)=e^{G(\theta) \cdot L A I / \cos \theta}$

where $P(\theta)$ is the gap probability in the observation zenith angle $\theta, G(\theta)$ is the leaf projection coefficient, and $\cos (\theta)$ is used for considering the path length.

We assume that the height is $H$, the path length that passes through the stand is $\mathrm{H} / \cos (\theta)$, and its representative projected area is $H \cdot \tan (\theta)$ (Fig. 1a) (Nilson, 1999). For a forest stand, the LAIs calculated in different zenith angles are comparable and compatible because the canopy is continuous, although the representative projected areas in different zenith angles are different. The leaf area per unit ground calculated in different zenith angles can be regarded as similar because the leaf area increases with the representative projected area. The measurement of different zenith angles can be regarded as different samples that represent the stand. Notably, the continuous canopy is an assumption in nearly all indirect LAI measurement methods, including the traditional clumping correction, because all of these methods use Eq. (1) or $\cos (\theta)$ for path-length correction.

\subsection{Beer's law for a single tree}

However, the LAIs calculated using Eq. (1) in different zenith angles for a single tree are incomparable and incompatible because the representative projected area changes, whereas the total leaf area remains unchanged (Fig. 1b). Therefore, these LAIs are ambiguous and cannot be averaged directly.

The use of leaf area density is suggested because the LAI of an individual tree is ambiguous, unless the size and position of the ground 
(a) Forest stand: $\operatorname{LAI}\left(\theta_{l}\right) \approx \operatorname{LAI}\left(\theta_{2}\right)$

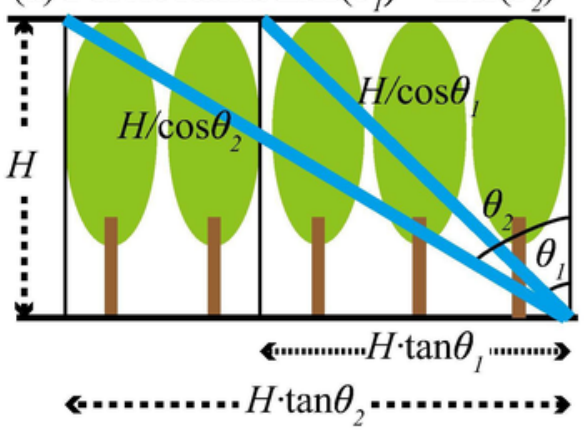

(b) Single tree: $\operatorname{LAI}\left(\theta_{1}\right) \neq \operatorname{LAI}\left(\theta_{2}\right)$

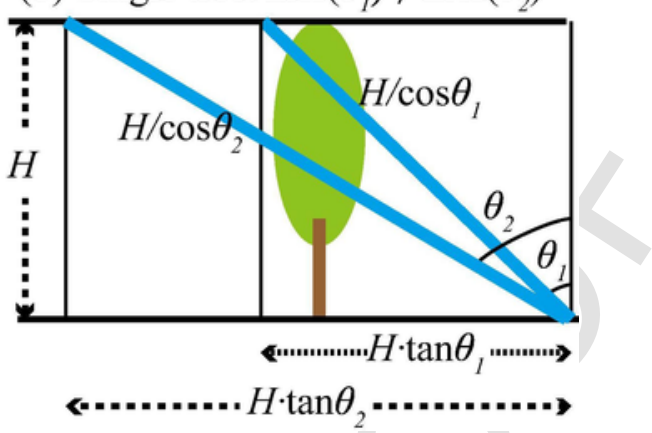

Fig. 1. Illustration of the Beer's law-based method for a forest stand (a) and for a single tree (b).

area are also provided (Li-COR, 2011). However, the leaf area density alone remains insufficient for characterizing a tree crown because its leaf area still varies within the volume. The total leaf area may be the optimum variable to characterize an individual tree because the area is fixed and independent. The leaf area density for the leaf area estimation is more closely related to Beer's law than the leaf area, but the volume is necessary to fix the leaf area expressed as follows:

$L A=\rho \cdot V$

where LA is the leaf area of a tree, $\rho$ is the leaf area density, and V is the volume of the tree crown.

The path length distribution model is modified to model the leaf area density of an individual tree by introducing a tree crown envelope with a known volume for the leaf area calculation.

\subsection{Path length distribution model for a single tree}

The path length distribution model was proposed to consider a crown shape-induced clumping effect within crowns by introducing the path length distribution into the theoretical prototype of Beer's law. Foliage clumping, that is, nonrandom foliage distribution, is a description of the spatial distribution of leaves. The path length distribution describes the thickness distribution of crowns, which are filled with leaves; the thickness distribution describes the spatial distribution of leaves. The path length distribution becomes the main factor for describing the spatial distribution of leaves by assuming a random foliage distribution within the crowns.

The path length distribution model was originally developed in traditional optical instruments, which can only obtain the relative path length distribution from the gap distribution data rather than the absolute one. The relative path length distribution and relative leaf area density are used as intermediate variables, which are sufficient for the LAI estimation in a forest stand; however, an absolute leaf area density is unavailable, which limits its use for LAI estimation for an individual tree.

The TLS provides the 3D point cloud data, which facilitate in acquiring the absolute path length distribution directly and consequently enable the path length distribution model to separate the path length and leaf area density. In this context, the path length distribution model is modified for an individual tree, which is expressed as follows:

$P=\int_{0}^{l_{\max }} e^{-G \cdot \rho \cdot l} \cdot p_{l}(l) d(l)$

where $P$ is the average gap probability in the tree crown, $G$ is the leaf projection function, $\rho$ is leaf area density, $l$ is the path length, and $p_{l}(l)$ is the path length distribution function, where $\int_{0}^{l_{\max }} p_{l}(l) d(l)=1$ . Furthermore, $P$ and $G$ are the basic inputs for the Beer's law-based method, and $p_{l}(l)$ can be calculated on the basis of the intersections between the laser pulses and the 3D tree crown envelope constructed from point cloud data. Detailed calculations will be discussed in the method section. The leaf area density can be retrieved with all the known variables using a root-finding algorithm.

\section{Materials}

\subsection{Study site}

The study site is located in the historical garden of the University of Strasbourg (Fig. 2) (48 $\left.35^{\prime} 4^{\prime \prime} \mathrm{N}, 7^{\circ} 45^{\prime} 49^{\prime \prime} \mathrm{E}\right)$. This experiment is a part of a long-term fieldwork experiment to measure and monitor the urban climate of the city of Strasbourg, particularly focusing on the role of vegetation in micro-climatic conditions (Najjar et al., 2015). The historical garden is a park with two rows of regularly spaced silver linden trees surrounded by $20 \mathrm{~m}$-high university buildings. This garden is a typical urban environment where trees are isolated but are commonly blocked by buildings and other trees from different directions. Each silver linden tree is approximately $9 \mathrm{~m}$ high with an under-crown height of approximately $2 \mathrm{~m}$. The DBH is approximately $0.3-0.4 \mathrm{~m}$, and the crown width is approximately $5 \mathrm{~m}$. The foliage of a silver lime tree appears in April and remains until October. The data acquired during the leafy and leafless (branches alone) periods from 2013 to 2017 were used to characterize the tree structures.

\subsection{TLS data}

The TLS data were collected through a time-of-flight laser scanner (Leica ScanStation C10) on July 3, 2013, and a phase-shift laser scanner (FARO Focus 3D X330) on January 25 and November 15, 2015; June 27, 2016; and July 6, 2017. At least seven stations were used in each experiment, where at least four stations have a complete view of the studied tree (Fig. 3). The acquisition parameters were selected on the basis of the time and data amount (Table 1). Several target spheres were adequately positioned in the field before scanning, and point clouds acquired from different stations were registered into the same coordinate system based on these target spheres. For each station, the point cloud was exported to PTX format, which contains the scanner location and information (coordinates and intensity) of all emitted laser pulses regardless whether the pulses obtain a return or not.

\subsection{Allometric data}

The allometric measurements of the leafy shoots were performed during the leafy period. Approximately 25 shoots of different lengths were sampled in each measurement to represent the existing shoot length. For each shoot, the shoot length, the number of leaves, the distance between leaf nodes, and the petiole length were measured manu- 


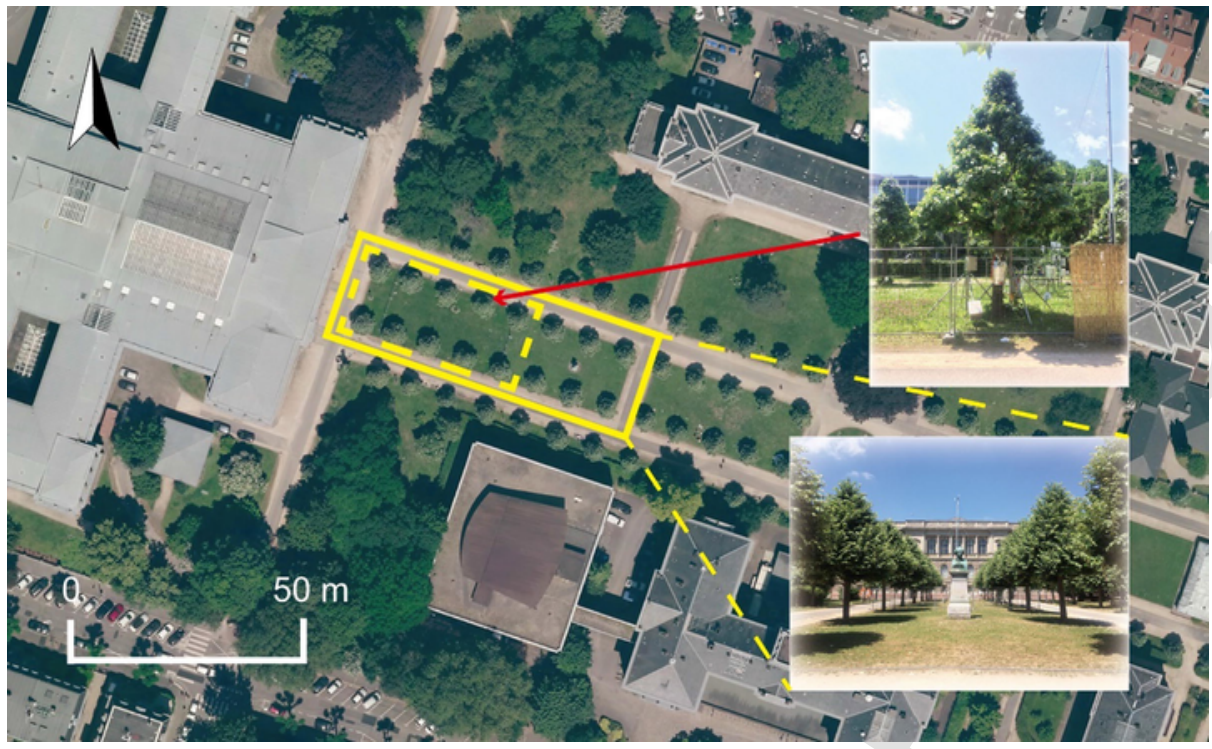

Fig. 2. Overview of the studied tree in the historical garden of the University of Strasbourg, France.

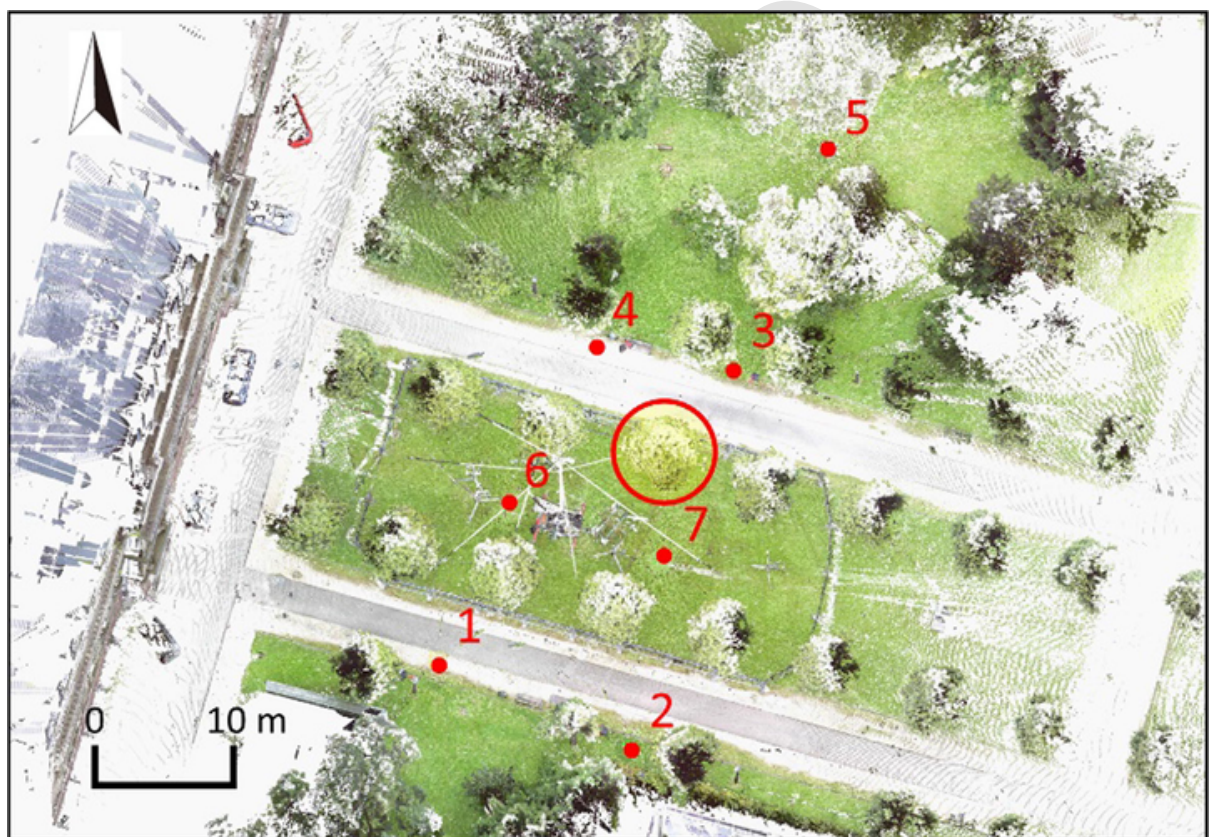

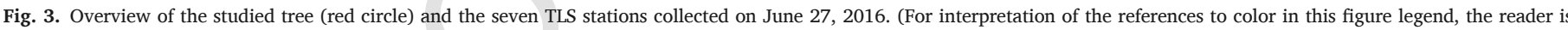
referred to the web version of this article.)

Table 1

Technical parameters of the two TLS systems.

\begin{tabular}{lll}
\hline Instrument & $\begin{array}{l}\text { Leica Scan Station } \\
\text { C10 }\end{array}$ & $\begin{array}{l}\text { FARO Focus 3D } \\
\text { X330 }\end{array}$ \\
\hline Ranging method & Time of flight & $\begin{array}{l}\text { Phase-shift } \\
\text { Resolution }\end{array}$ \\
Spacing between points & $0.2 \mathrm{mrad}$ & $0.6 \mathrm{mrad}$ \\
Beam divergence & $\sim 2 \mathrm{~mm}$ at $10 \mathrm{~m}$ & $0.19 \mathrm{mrad}$ \\
Beam waist diameter $(1 /$ & $0.1 \mathrm{mrad}$ & $2.25 \mathrm{~mm}$ \\
e) & $2.5 \mathrm{~mm}$ & \\
\hline
\end{tabular}

ally using a measuring tape. All leaves were cut and placed on a piece of red paper with a reference square of $4 \mathrm{~cm} \times 4 \mathrm{~cm}$, and then the area of each leaf was extracted through an automatic image processing system. On the basis of these measurements, the allometric statistics of the leaf surface area, number of leaves, and shoot length were established (Sonohat et al., 2006) of (Fig. 4).

Similar allometric statistics were obtained from four dates: August 2013, September 2014, July 2017, and August 2017. These allometric statistics were used for a reliable estimation of the total leaf area of a tree if the lengths of all its shoots are known. The skeletons of all the shoots of the studied tree were reconstructed on the basis of the 3D point cloud to obtain a complete tree structure and length of all shoots. In 2013, the shoots were reconstructed through manual digitalization, which takes three months. Although the manual digitalization is very time consuming, it provides an accurate reference defining the real geometry of the studied tree. For other years, the shoots were reconstructed through an automatic 3D reconstruction using a Boudon et al. (2014) method (Bournez et al., 2017) (Fig. 5). Then, the length of each reconstructed shoot was obtained and used for the leaf area calculation 


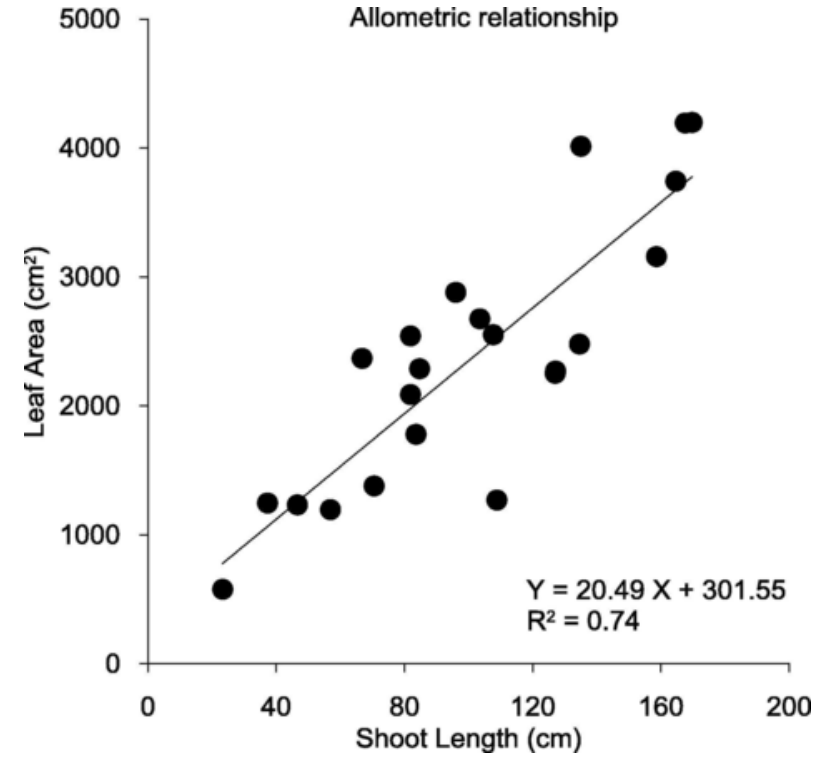

Fig. 4. Allometric relationship between the leaf area and shoot length.

based on the allometric statistics. The total leaf area obtained in the summer of 2013 was considered the most accurate because the allometric statistics and shoot lengths were measured manually. The leaf areas of the studied tree were $187.39,182.47$, and $179.86 \mathrm{~m}^{2}$ on July 3,2013 ; June 27, 2016; and July 6, 2017, respectively. The three measurement dates were similar with a difference of approximately a week, and their results were proximate. Therefore, we consider these results reasonable and regard them as the reference data in the present study.

\subsection{Leaf angle distribution measurement}

The leaf angle was obtained from the leaf normal distribution computed based on the acquired high-density point cloud and open-source software CloudCompare (www.cloudcompare.org). The trunk and branches were removed before computation. The leaf normals were computed using a plane surface model (Bailey and Mahaffee, 2017). The computed leaf inclination angles were validated through a method proposed by Ryu et al. (2010) and Pisek et al. (2011); this method measures the selected leaves that are oriented nearly parallel to the viewing direction. The point clouds were visualized and inspected in CloudCompare using a leveled camera setting. The results showed that the computed leaf inclination angles that use CloudCompare agree well with those measured manually. The leaf projection function $G$ is expressed as follows:

$G(\theta)=\int_{0}^{\pi / 2} A\left(\theta, \theta_{l}\right) g_{l}\left(\theta_{l}\right) d\left(\theta_{l}\right)$

$A\left(\theta, \theta_{l}\right)=\left\{\begin{array}{cc}\cos \theta \cos \theta_{l}, & \left|\cot \theta \cot \theta_{l}\right|>1 \\ \cos \theta \cos \theta_{l}[1+(2 / \pi)(\tan \psi-\psi)], & \left|\cot \theta \cot \theta_{l}\right| \leqslant 1\end{array}\right.$

where $\psi=\cos ^{-1}\left(\cot \theta \cot \theta_{l}\right), \theta$ is the viewing zenith angle, and $\theta_{l}$ is the leaf zenith angle.

The leaf projection functions computed from the point cloud of each station and the merged point cloud of four stations were compared, and a minimal difference $(<3 \%$ ) was found (Fig. 6 ). The $G$ function of the merged point cloud was used for further calculation.

\section{Methods}

The leaf area density was estimated using the path length distribution and the gap probability within the studied tree crown using Eq. (3). The leaf projection function $G$ was calculated from the leaf angle distribution measured in the high-density point cloud. An envelope was reconstructed for the path length distribution calculation, and a tree crown mask was used to limit the calculation within the studied tree crown. The envelope was reconstructed on the basis of the merged point cloud obtained from multiple stations, whereas the tree crown mask, the path length distribution, the gap probability, and the leaf area density were calculated for each station separately.

\subsection{Envelope reconstruction}

Point cloud data from multiple stations were merged together to characterize the complete envelope of each tree. Then, the crown of the studied tree was segmented manually through CloudCompare. An alpha shape algorithm (Edelsbrunner and Mücke, 1994) was then applied to the segmented point cloud to reconstruct the envelope of the tree crown by using MATLAB. This algorithm can reconstruct convex and concave envelopes (Fig. 7). Convex envelope, which is composed of hundreds of triangular facets, is the smallest convex region that encloses all points, whereas concave envelope, which is composed of thousands of triangular facets, fits the tortuosity of the tree crown well. In the concave envelope, the outline was refined by removing the large gaps in the edges. In the present study, convex and concave envelopes were tested.

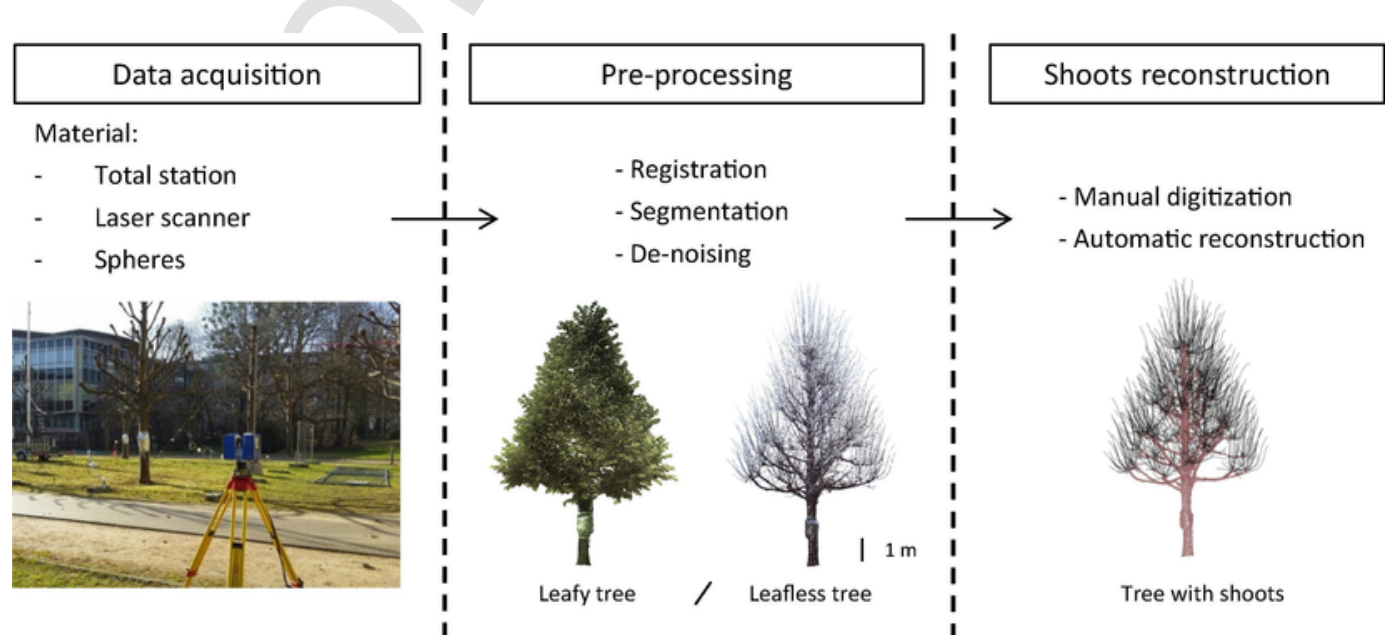

Fig. 5. TLS data acquisition, pre-processing, and reconstruction of the studied tree. 


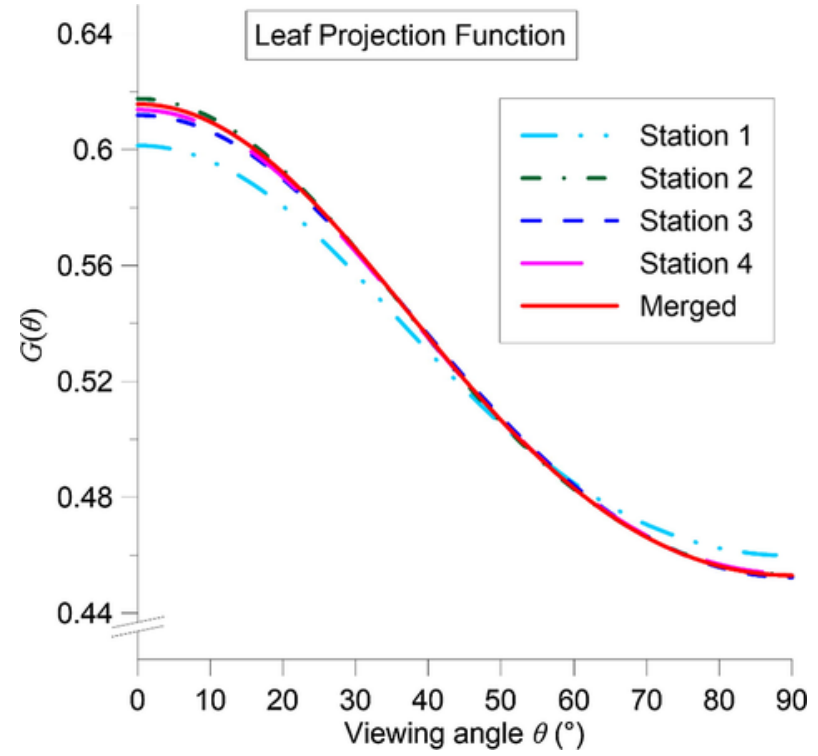

Fig. 6. Leaf projection functions $(G)$ computed from the point cloud of each station and the merged point cloud of four stations acquired on July 3, 2013.

\subsection{Tree crown mask}

For each station, a tree crown mask was used to filter the laser pulses from entering the tree crown because these pulses did not contribute to the retrieval of within-crown leaf area. Intersections were calculated between the tree crown envelope of the studied tree and each emitted laser pulse regardless whether the pulse obtain a return or not (Fig. 8). The laser pulses without any intersections with the envelope were filtered (Fig. 8, laser pulse a). Meanwhile, the pulses with intersections but were blocked by objects between the studied tree and the laser scanner (Fig. 8 , laser pulse b) also did not contribute to the retrieval. Thus, these laser pulses were filtered by distance. If the distance from the laser scanner is shorter to the return than to the envelope, then this result indicated that the pulse is blocked by other objects and thus does not reach the envelope. All further processes only include the laser pulses within the tree crown mask, which is composed of emitted laser pulses (Fig. 8c, d, and e).

The laser pulses without returns should be considered additionally because wrong returns associated with mixed pixels are removed before outputting data, which causes an underestimation of the number of returns (Pimont et al., 2015). Specifically, the coordinates of laser pulses without returns are $(0,0,0)$ in the PTX file; the direction of the laser pulse should be identified to determine whether the pulse has intersections with the envelope or not. The direction of the TLS could be interpolated accurately because the TLS acquires data with a consistent zenith and azimuth angular spacing. The data in the PTX file were converted as an image, and each record in the PTX file could be regarded as a pixel in the image. First, we calculated the zenith and azimuth angles of the pixels with known coordinates. Second, the zenith and azimuth angles of the remaining pixels were interpolated on the basis of the zenith and azimuth angles of the surrounding pixels and the zenith and azimuth angular spacing of the TLS (resolution in Table 1). The directions of these laser pulses could be used for the tree crown mask and path length distribution calculation similar to the other laser pulses with known coordinates once these directions were identified.

\subsection{Path length distribution}

The path length distribution was calculated for each station using the tree crown mask. The path length represents the distance a laser pulse travels through the studied tree crown. Each laser pulse is a ray emitted from the TLS and is considered as having an infinite length. The intersections of each laser pulse with each triangular facet of the tree crown envelope were then calculated. If a laser pulse encounters the tree crown envelope, then the pulse demonstrates two intersections, that is, one entering and the other exiting the envelope. Hence, the path length was calculated as the distance between the two intersecting points. Notably, the emitted laser pulses with a return within the envelope (Fig. 8, laser pulse c) also used this method for path-length calculation, although the pulse does not actually pass through the envelope. Finally, the path length distribution was obtained from the statistics of all the path lengths.

\subsection{Gap probability}

The gap probability was calculated from the TLS data of each station separately using the tree crown mask. Leica C10 and FARO Focus 3D X330 are single-return TLSs; therefore, the gap probability of the studied tree was calculated as the ratio of the number of laser pulses passing through and those entering the tree crown. In addition, the surrounding objects should be considered for a single tree compared with a forest stand. First, the tree crown mask was used to calculate the number of laser pulses that enter the tree crown; therefore, the laser pulses that are blocked by instruments or other trees are omitted from the calculation (Fig. 8, laser pulse b). Second, the laser pulse with or
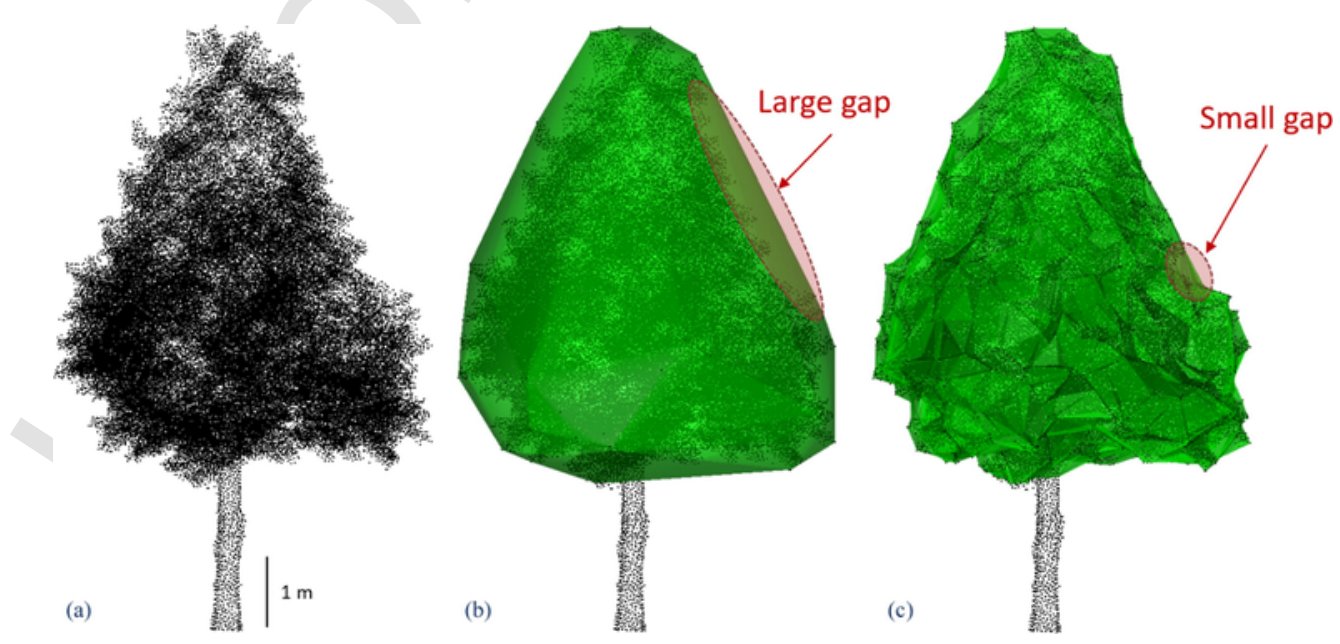

Fig. 7. Merged point cloud (a), convex envelope (b), and concave envelope (c) of the studied tree crown. 


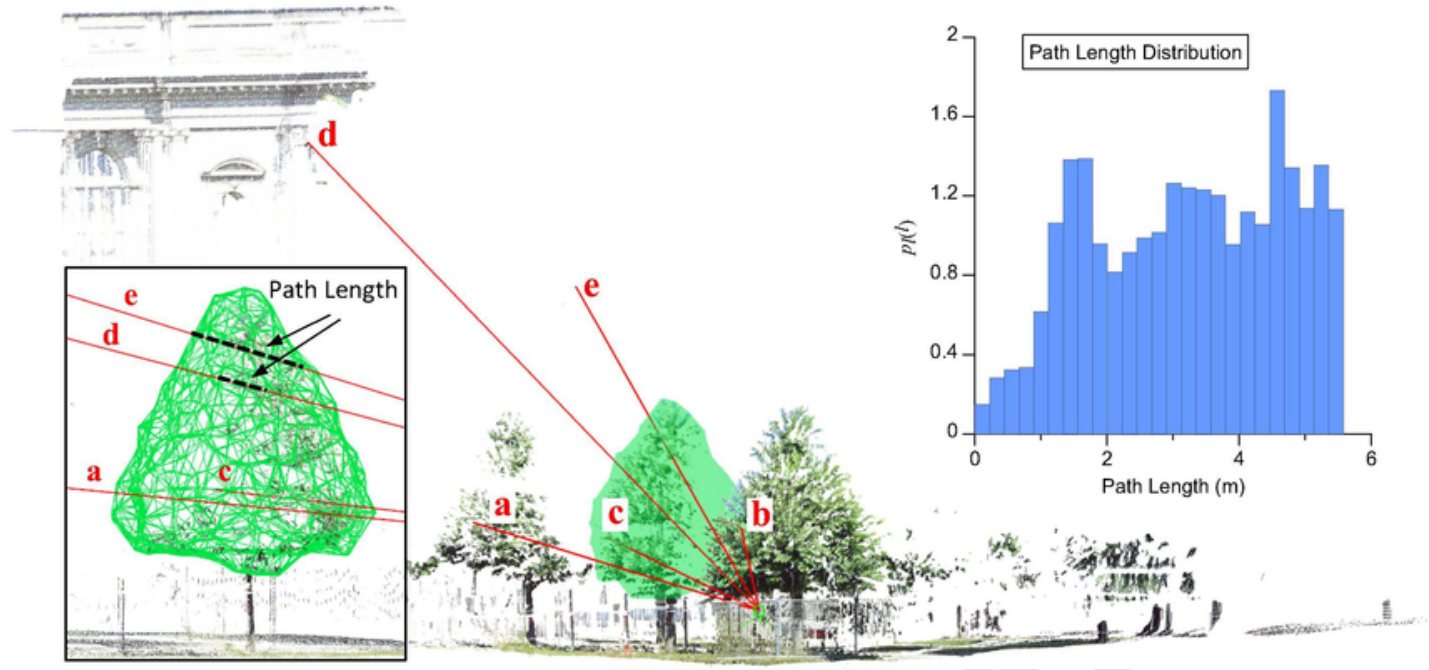

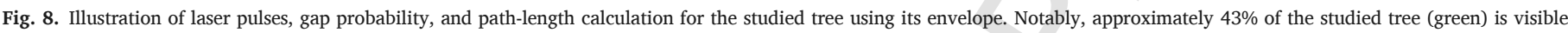

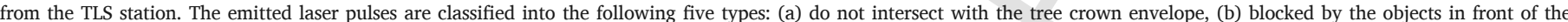

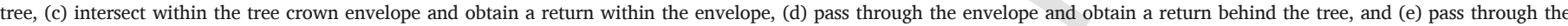

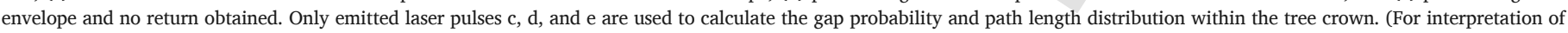
the references to color in this figure legend, the reader is referred to the web version of this article.)

without a return from the object behind the studied tree was regarded as a gap (Pimont et al., 2015) (Fig. 8, laser pulse d). However, the laser pulses, which returns were farther than the envelope, were also considered gaps for the tree crown in addition to the laser pulses without returns.

\subsection{Weighted mean of the multi-station leaf area density}

The studied tree was scanned from several stations (Fig. 3), and the leaf area density was calculated from each station for the same tree separately using the path length distribution model (Eq. (3) with the root-finding algorithm. Specifically, we selected Brent's method for the GNU Scientific Library to be the root-finding algorithm in our programming, and this method can solve the leaf area density of a tree within a few seconds. Different stations have various distances from the studied tree and fields of view of the studied tree. We averaged the leaf area densities that were calculated from different stations with weighting factors to use additional samples and achieve a reliable result. The weighted mean and standard deviation of the leaf area density are expressed as follows:

$\bar{\rho}_{w}=\frac{\sum_{i=1}^{N} \rho_{i} \cdot w_{i}}{\sum_{i=1}^{N} w_{i}}$

$\sigma_{w}=\sqrt{\frac{\sum_{i=1}^{N}\left(\rho_{i}-\bar{\rho}_{w}\right)^{2} \cdot w_{i}}{\sum_{i=1}^{N} w_{i}}}$

where $\bar{\rho}_{w}$ is the weighted mean of the leaf area density, $\sigma_{w}$ is the weighted standard deviation, $\rho_{i}$ is the leaf area density that was calculated from each station, and $w_{i}$ is the weighting factor. The number of laser pulses and the sum of path lengths were tested as the weighting factors. The number of laser pulses is related to the number of samples in the scanning direction, whereas the sum of the path lengths contains the additional depth information and is related to volume proportion.

\subsection{Leaf and woody areas}

The allometric method provided leaf area directly because the allometric statistics are established between leaf area and shoot length.
However, the gap probability-based method provided the sum of leaf area and woody area because both of leaf and woody components blocked the laser beam during the leafy period. We converted their results and ensured that they provide the same physical meaning to compare the results of the allometric and gap probability-based methods.

For the gap probability-based method, the data acquired during the leafless period (branches alone) were used to quantify the woody area using the same model used during the leafy period. The leaf area of the gap probability model is expressed as follows:

$L A=P A-W A$

where $L A$ is the leaf area; $P A$ is the plant area, which is the result of the gap probability-based method during the leafy period; and $W A$ is the woody area, which is the result of the gap probability-based method during the leafless period.

\section{Results}

\subsection{Leaf area density retrieval from different stations using the path length distribution model}

In each measurement, the leaf area density is retrieved from different TLS stations separately (Table 2), and the weighted mean of the different stations is calculated using Eq. (6). Stations 3, 4, 6, and 7 are in the range of 7.2-11.2m away from the studied tree, and no object occludes the scanning (Fig. 3). Approximately $100 \%$ of the studied tree can be observed from these stations, and a large number of laser pulses reach the tree crown. The path length distributions obtained from the four stations are similar (Fig. 9). The results of Stations 3, 4, 6, and 7 are closest to the weighted mean of the seven stations. Stations 1 , 2, and 5 are not set specifically for the studied tree but for the whole garden. The three stations are farther away from the studied tree than the other stations and only $9-27 \%$ of the studied tree is visible from them because the field of view of the TLS is blocked by other trees or objects. The path length distributions of the three stations vary and are different from the other stations (Fig. 9) because different parts of the tree crown are observed. Stations 1,2 , and 5 have a small number of laser pulses that enter the tree crown; the number of laser pulses is only approximately $5 \%$ of that of another station. The difference is larger between the weighted mean and the result of Station 1 or 2 than 


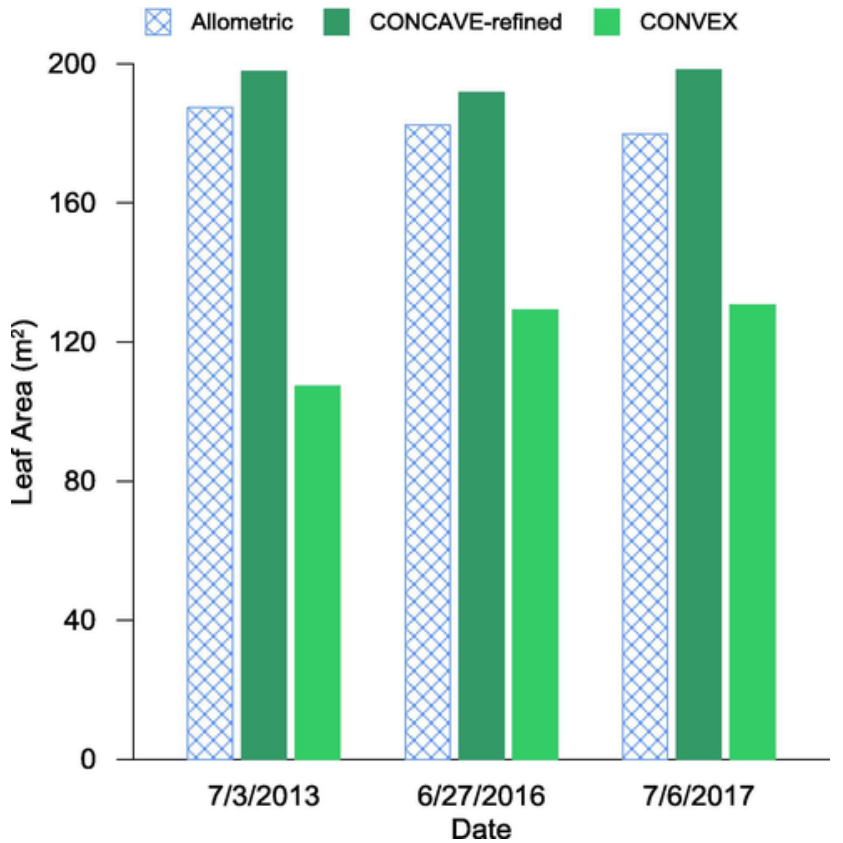

Fig. 11. Comparision of the retrieved leaf area using the TLS data and path length distribution model with reference measurement for an individual tree. The refined concave and convex envelopes are tested.

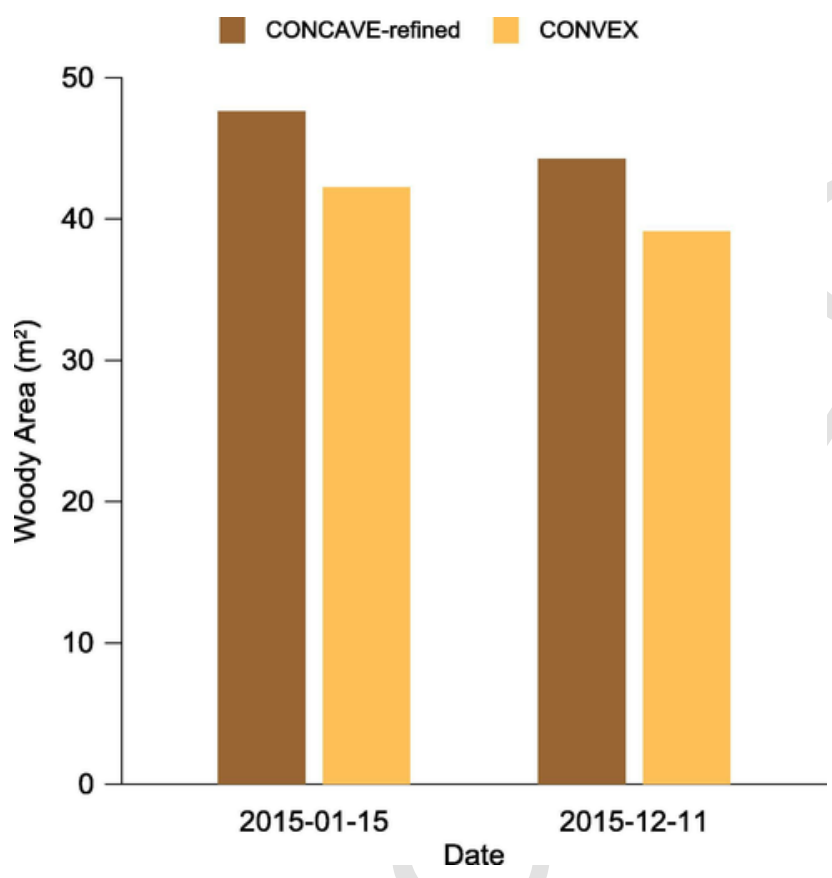

Fig. 12. Comparison of the woody area retrieved using the refined concave and convex envelopes.

27, 2016, from seven stations (Table 3). The results showed that the proposed method can characterize different plant areas with low standard deviation despite the absence of an allometric measurement for the ten trees. Seven results for each tree were retrieved separately from seven TLS stations at different distances. The plant areas of the ten trees ranged from $184.38 \mathrm{~m}^{2}$ to $234.21 \mathrm{~m}^{2}$. The relative proportion of the weighted standard deviation to the weighted mean of the seven re-
Table 3

Plant area of the ten trees retrieved from the seven TLS stations on June 27, 2016.

\begin{tabular}{llll}
\hline $\begin{array}{l}\text { Tree } \\
\text { number }\end{array}$ & $\begin{array}{l}\text { Distance } \\
(\mathrm{m})\end{array}$ & Plant area $\left(\mathrm{m}^{2}\right)$ & \\
\hline & & $\begin{array}{l}\text { Weighted } \\
\text { mean }\end{array}$ & $\begin{array}{l}\text { Weighted standard } \\
\text { deviation }\end{array}$ \\
\hline 1 & & 186.10 & $10.03(5.39 \%)$ \\
2 & $14.9-37.8$ & 192.97 & $17.43(9.03 \%)$ \\
3 & $9.1-33.2$ & 184.38 & $11.01(5.97 \%)$ \\
4 & $5.9-29.0$ & 234.21 & $11.41(4.87 \%)$ \\
5 & $7.2-25.9$ & 225.82 & $28.07(12.43 \%)$ \\
6 & $8.9-26.6$ & 184.41 & $18.62(10.10 \%)$ \\
7 & $13.5-46.5$ & 197.83 & $27.72(14.01 \%)$ \\
8 & $8.5-42.6$ & 189.22 & $9.90(5.23 \%)$ \\
9 & $5.4-39.3$ & 228.49 & $15.35(6.72 \%)$ \\
10 & $5.3-37.6$ & 231.14 & $38.63(16.71 \%)$ \\
\hline
\end{tabular}

sults for the ten trees ranged from $4.8 \%$ to $16.7 \%$, thereby indicating that the proposed method is generally stable for plant area estimation.

\subsection{Leaf area retrieved using TLS data at different resolutions}

To analyze the influence of scanning resolution on leaf area estimation, the TLS data on July 3, 2013, with an angular resolution of 0.2 mrad were sampled every $2,3,5,10,15$, and 20 points to build the TLS dataset with resolutions of $0.4,0.6,1,2,3$, and $4 \mathrm{mrad}$, respectively. The same method and process were applied to the TLS data to estimate the leaf area at different resolutions. Therefore, the differences of the leaf area only resulted from the different scanning resolutions (Fig. 13). The leaf areas at different resolutions were generally consistent, with a maximum difference of $6.6 \%$ between 0.2 and 4 mrad. The leaf area, as well as its weighted standard deviation, slightly increased with the decrease in the resolution. The gap probability of the studied tree crown decreased with the decrease in the resolution. The increase of the leaf area is generally correlated with the decrease in the gap probability because the small gap probability corresponds to a large leaf area.

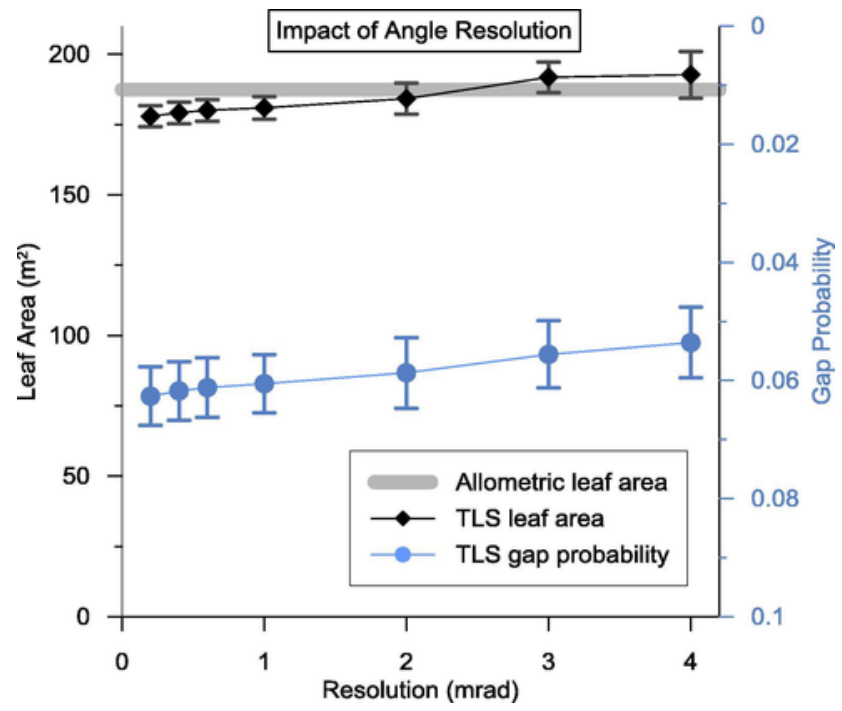

Fig. 13. TLS-derived leaf area and gap probability of the studied tree crown at different angular resolutions. The multi-station results retrieved using the path length distribution model are the weighted mean. The error bars denote the weighted standard deviation of the multi-station results. 


\section{Discussion}

\subsection{Consistency of using the leaf area density and path length distribution}

The results of different stations were consistent, especially when samples are sufficient (Table 2), possibly because large visible proportions and number of pulses correspond to a high confidence result. The results of Stations 1 and 2 relatively differed compared with the weighted mean because the two stations are distant from the studied tree, and their fields of view are blocked by other trees. The number of laser pulses that reach the studied tree was only approximately $5 \%$ higher from Station 1 or 2 than from the other stations, thus resulting in an inadequate sampling and low reliability result. The contribution of Station 1 or 2 was only approximately $1 \%$ after introducing the number of pulses as a weighting factor, and its uncertainty to the weighted mean was largely limited.

The relative proportion of standard deviation to the weighted average of the leaf area $(1.5 \%-3.8 \%)$ was found to be smaller than that of gap probability $(7.9 \%-11.2 \%)$ (Fig. 13), thereby indicating that the uncertainty is reduced after using the path length distribution model. This result is due to gap probability varies with path length distribution, which also varies at different parts of the tree crown scanned from the different stations (Fig. 9). The use of path length distribution compensated the difference in gap probabilities in different parts, provided a relatively consistent leaf area, and eliminated the limitation of observation zenith angle. Traditional methods generally do not use the data at zenith angles larger than $60^{\circ}$ given the limitation of data acquisition and quality (Demarez et al., 2008; Gonsamo and Pellikka, 2009). The observations at a large zenith angle of approximately $90^{\circ}$, which cannot be used in the traditional methods using Eq. (1) because $1 / \cos \left(90^{\circ}\right)$ is undefined, could be used in the path length distribution model (Table 2). The TLS laser pulses at zenith angles of approximately $90^{\circ}$ frequently represented a large proportion of the emitted laser pulses given the relative position and height between the TLS station and the tree crown (Table 2). The use of the data of approximately $90^{\circ}$ is particularly important because most of the leaves are distributed at the bottom of the tree crown.

\subsection{Advantage of the weighted mean of different stations for the leaf area estimation}

The leaf area density means weighted by the number of pulses and by the sum of the path lengths were considerably close, thus indicating that either the number of pulses or the sum of path lengths is a favorable weighting factor (Table 2). The use of weighted mean demonstrates the following advantages despite the feasibility of filtering the data with low reliability: consistency, universality, and efficiency. First, the same TLS dataset can be used for each tree in the study area, thereby ensuring a consistent input for the leaf area estimation. Second, no subjective process or threshold is required for filtering the bad data, thus ensuring that all the calculations are quantitative and universal. Therefore, we considered the results of all stations, although several of these results would enlarge the weighted standard deviation. Third, using the weighted mean eliminates the requirement of several complete scans for each tree separately, thereby largely reducing the required scanning stations. The weighted mean method uses the data of all stations, regardless of the visible proportion. The only necessary input is a complete tree crown envelope, which is not sensitive to the point density. If the merged point cloud can characterize the envelope of a tree crown, then the method will provide a leaf area and a weighted standard deviation to indicate its uncertainty. The method is particularly useful for measuring the leaf area of several trees because only several shared stations with sufficient coverage of all the studied trees are required rather than several stations for each tree separately.

\subsection{Influence of the TLS laser beam size on leaf area estimation}

The finite size of the TLS laser beam is reported to underestimate the canopy gap probability (Beland et al., 2011). The accurate gap probability is supposed to be the proportion of the laser beams, which centers do not fall within the area of the leaves. However, a laser beam has a finite diameter. The laser beams, which edges hit the leaf area with its center outside the leaf area, may also obtain certain returns. These returns are misclassified as a canopy and underestimate the proportion of the gaps. Beland et al. (2011) proposed a method to correct the gap probability estimation by accounting for the laser beam size. The results show that a beam centered outside the leaf area with an $800 \mathrm{~m}$ long-range TLS (ILRIS-3D), which has a laser beam diameter of $7.7 \mathrm{~mm}$ at a $20 \mathrm{~m}$ distance, but at a distance smaller than $2.3 \mathrm{~mm}$ from its edge will trigger a return on the side of an elliptical leaf; the gap probability is reported to be underestimated by approximately $20 \%$ from the zenith angle of $40-90^{\circ}$. This correction requires the accurate value of minimum intensity required to trigger a return and the radius of the parabolic mirror; these pieces of information are unavailable in the technical documents of the TLS used in this study. Therefore, we conducted a relative analysis of the effect of the laser beam size on the gap probability estimation. In this study, we used a $330 \mathrm{~m}$-range TLS (FARO X330), which has a laser beam diameter of $4.15 \mathrm{~mm}$ at a $20 \mathrm{~m}$ distance. First, the ranging distance represents the capability of the TLS to trigger a return. A $330 \mathrm{~m}$-range TLS is less likely to trigger a partial return because its capability of triggering a low signal is approximately $41.25 \%$ compared with using an $800 \mathrm{~m}$ long-range TLS. Second, the laser beam diameter in this study was $4.15 \mathrm{~mm}$, which is $53.9 \%$ higher than that of the previous study. Therefore, the maximum distance between the laser beam center and the leaf edge for triggering a return should be less than $2.3 \mathrm{~mm} \times 53.9 \%=1.24 \mathrm{~mm}$. In addition, the mean leaf area in this study is $150-250 \mathrm{~cm} 2$, which is four times higher than that of the previous study. Taking all factors into consideration, the gap probability underestimation caused by laser beam size is less than $5 \%$ based on the method of Beland et al. (2011).

\subsection{Influence of the TLS scanning resolution on leaf area estimation}

The TLS scanning resolution influences leaf area estimation in two aspects, namely, gap probability and path length distribution. The path length distribution is decided by the envelope, which is not sensitive to the TLS resolution. The high resolution results in a dense point cloud and can characterize the inner details of the studied tree well but slightly influences the outline of the tree, which is constantly optimally scanned. The envelopes reconstructed from the TLS data at different resolutions show nearly no difference.

The similar trends of the increase in the leaf area and decrease in the gap probability at different resolutions indicate that the TLS resolution mainly influences the leaf area estimation by influencing the gap probability (Fig. 13). The TLS scanning resolution corresponds to the sampling density for the gap probability measurement. Theoretically, the gap probability will not change largely with the change in the resolution if samples are sufficient. In this study, the gap probability does not change largely but shows a decreasing trend, possibly due to the edge effect. A number of gaps at the edge of the tree crown mask are observed. The edge part will be less sampled, and these gaps will be less observed when the resolution becomes coarse. The weighted mean of the leaf area remains unchanged largely when the TLS angle resolution becomes coarse, whereas its weighted standard derivation increases (Fig. 13). The relatively minimal difference (6.6\%) between the results of 0.2 and $4 \mathrm{mrad}$ resolutions indicates that this method is not 
sensitive to the scanning resolution. However, a high resolution is still preferred to provide a minimal uncertainty of the leaf area estimation.

\subsection{Comparison of convex and concave envelopes}

The comparisons with the allometric measurement of an individual tree show that concave envelope performs better than convex envelopes, which considerably underestimate the leaf area. The concave envelope separates the tree crown and the gaps outside the tree crown well because the concave envelope it fits the tortuosity of the edge of the tree crown. However, the convex envelope is the smallest convex with not pit and thus contains many empty spaces at the edge. Therefore, the convex envelope contains additional gaps. The leaf area underestimation of the convex envelope is due to the large gaps in the edge of the tree crown; these large gaps significantly increase the total gap probability within the tree crown. The difference between the gap probability in the edge and within the tree crown is the typical clumping effect of Beer's law. The leaf area underestimation will be obvious when the difference is large. The gap probability within a tree crown is extremely small because the trees constantly have nearly the most leaves in the beginning of July. At this time, the gap probability in the edge is much larger than that within the tree crown. However, the underestimation becomes less noticeable in winter because the gap probability within the tree crown is also large (Fig. 12).

The vegetation heterogeneity within the crown, including the clumping of needles in the shoots of conifers (Stenberg, 1996a, 1996b) and non-uniform leaf area density within the crown, may lead to a relative underestimation of the leaf area. In this study, we selected the broadleaf trees to avoid the shoot clumping in conifers. In addition, we used the path length distribution to account the crown shape-induced within-crown clumping caused by inconsistent path length (Hu et al., 2014) while assuming the uniform leaf area density within the tree crown envelope. The uniform leaf area density assumption within the tree crown envelope was met in the studied trees during the growing season; thus, the results of this study are favorable. Accurate envelope reconstruction using a matrix of voxels (Beland et al., 2014)will be useful for complex tree structures and can be tested to improve the results in the future.

\subsection{Comparison of time-of-flight and phase-shift laser scanner on leaf area estimation}

The scanner laser sensors used in this study are based on discrete returns. As described by Newnham et al. (2012), the time-of-flight scanner emits a laser pulse and measures the discrete time-of-flight of a return echo from the intercepted target. A time-of-flight scanner provides a high accuracy at large range and resolves gaps well. A phase-shift scanner uses a constant wave laser with a certain frequency of intensity. The shifts in the phase of the returned modulations are used to determine a range. The phase-shift scanners can provide samples at much higher frequencies and are generally lighter and cheaper than the "equivalent" time-of-flight instruments. However, the data are noisy around the edges of objects and the gaps are relatively difficult to resolve. Limited results show that the performance of a time-of-flight laser scanner is more stable than the phase-shift laser scanner for the leaf area estimation because the weighted standard derivation of the leaf area retrieved in 2013 is smaller using a time-of-flight laser scanner than using a phase-shift laser scanner (Fig. 10). Processing point clouds obtained from the time-of-flight scanners is simple and robust because the laser pulses that intercept with the objects will provide accurate coordinates; thus, classifying the distinguished vegetation returns and gaps is easy. Meanwhile, the processing point clouds ob- tained from the phase-shift laser scanner is relatively complex because several partial beam interceptions (at the edge of the canopy components) lack coordinate information, although they have intensity information (Newnham et al., 2012). The directions of these records can be accurately calculated using the zenith and azimuth angle interpolations given the TLS scans in a specific order and angle interval. However, identifying whether such a leafy record is within the studied tree crown or in other trees is difficult because the distance cannot be accurately interpolated. Therefore, the results of the phase-shift laser scanner show significant uncertainties than the time-of-flight laser scanner on the leaf area estimation. However, the weighted mean of the leaf area retrieved using a phase-shift laser scanner is approximate to that retrieved using a time-of-flight laser scanner. Therefore, the phase-shift laser scanner is still useful considering its low instrument weight and high scanning speed.

\section{Conclusions}

A path length distribution model is modified to estimate the leaf area of an individual tree in urban areas. Accurate path lengths, which allow the path length distribution model to retrieve the leaf area density, rather than the relative path lengths in the original model, are directly obtained from the 3D TLS data. The path lengths are obtained by calculating the interceptions between all the emitted laser pulses and the tree envelope reconstructed from multi-station point clouds. Comparison with a reference tree crown reconstructed from digitizing all shoots manually and using allometric measurement shows that the TLS-retrieved leaf areas using the path length distribution model performs very well.

The new model eliminates the limitation of the observation zenith angle, is not limited by the surrounding buildings or other objects in urban areas, and can use the laser pulses of approximately $90^{\circ}$, which contain most of the leafy information given the relative position and height between the TLS station and the tree crown but cannot be used by traditional methods. The requirement of data amount and point density is largely relaxed, making leaf area estimation more efficient. In addition, the leaf area estimation using the TLS point cloud is not sensitive to scanning resolution.

The leaf areas of different stations are consistent, especially when samples are sufficient. The use of the weighted mean of leaf area estimations from different stations by the sample numbers further improves the universality and efficiency of the proposed method because the weighted mean can use the data from all stations even if occlusions exist.

The concave envelope performs better than the convex envelope because the large gaps in the edge were removed. This envelope is preferred during summer when the tree crown is dense and has few gaps. Further studies are required to analyze the influence of an envelope in other seasons. In this study, the leaves are generally uniformly distributed within the envelope and the discrepancy of leaf distribution is not considered, which might cause underestimation for trees with complex structures. An accurate envelope reconstruction that uses a matrix of voxels will be useful for describing complex tree structures. Existing data show that the performance of the time-of-flight laser scanner is more stable in the leaf area estimation, but the phase-shift laser scanner is lighter and faster. The comparison of time-of-flight and phase-shift laser scanners in one measurement can be a challenging strategy to analyze their differences quantitatively. Nearly all the data processing is automatic, except for the manual segmentation of the studied tree. The integration of the automatic segmentation algorithms into the calculation will be interesting for rapid leaf area extraction of multiple independent trees with several scanning stations in urban areas. 


\section{Acknowledgments}

This work was supported by the key program of the National Natural Science Foundation of China (NSFC) (Grant No. 41331171), the National Basic Research Program of China (Grant No. 2013CB733402), and the China Scholarship Council. The authors also thank the Interreg Atmo-IDEE program and the AVENUE project of the CNES/TOSCA, France, for the financial support and for allowing the researchers to use the historical garden of the University of Strasbourg. The authors also would like to thank the anonymous reviewers for their helpful and valuable comments that have greatly improved the paper.

\section{References}

Alonzo, M., Bookhagen, B., McFadden, J.P., Sun, A., Roberts, D.A., 2015. Mapping urban forest leaf area index with airborne lidar using penetration metrics and allometry. Remote Sens. Environ. 162, 141-153.

Bailey, B.N., Mahaffee, W.F., 2017. Rapid measurement of the three-dimensional distribution of leaf orientation and the leaf angle probability density function using terrestrial LiDAR scanning. Remote Sens. Environ. 194, 63-76.

Beland, M., Baldocchi, D.D., Widlowski, J.L., Fournier, R.A., Verstraete, M.M., 2014. On seeing the wood from the leaves and the role of voxel size in determining leaf area distribution of forests with terrestrial LiDAR. Agr. Forest Meteorol. 184, 82-97.

Beland, M., Widlowski, J.L., Fournier, R.A., Cote, J.F., Verstraete, M.M., 2011. Estimating leaf area distribution in savanna trees from terrestrial LiDAR measurements. Agr Forest Meteorol. 151, 1252-1266.

Benjamin, M.T., Winer, A.M., 1998. Estimating the ozone-forming potential of urban trees and shrubs. Atmos. Environ. 32, 53-68.

Boudon, F., Preuksakarn, C., Ferraro, P., Diener, J., Nacry, P., Nikinmaa, E., Godin, C., 2014. Quantitative assessment of automatic reconstructions of branching systems obtained from laser scanning. Ann. Bot-London 114, 853-862.

Bournez, E., Landes, T., Saudreau, M., Kastendeuch, P., Najjar, G., 2017. From TLS point clouds to 3D models of trees: a comparison of existing algorithms for 3D tree reconstruction. ISPRS-Int. Arch. Photogram. Remote Sens. Spat. Inform. Sci. 42, 2.

Bouvier, M., Durrieu, S., Fournier, R.A., Renaud, J.P., 2015. Generalizing predictive models of forest inventory attributes using an area-based approach with airborne LiDAR data. Remote Sens. Environ. 156, 322-334.

Chen, J.M., 1996. Optically-based methods for measuring seasonal variation of leaf area index in boreal conifer stands. Agr. Forest Meteorol. 80, 135-163.

Chen, J.M., Cihlar, J., 1996. Retrieving leaf area index of boreal conifer forests using landsat TM images. Remote Sens. Environ. 55, 153-162.

Chianucci, F., Puletti, N., Giacomello, E., Cutini, A., Corona, P., 2015. Estimation of leaf area index in isolated trees with digital photography and its application to urban forestry. Urban For. Urban GREE 14, 377-382.

Colaizzi, P.D., Evett, S.R., Brauer, D.K., Howell, T.A., Tolk, J.A., Copeland, K.S., 2017. Allometric method to estimate leaf area index for row crops. Agron. J. 109, 883-894.

Daughtry, C.S.T., 1990. Direct measurements of canopy structure. Remote Sens. Rev. 5, 45-60.

Demarez, V., Duthoit, S., Baret, F., Weiss, M., Dedieu, G., 2008. Estimation of leaf area and clumping indexes of crops with hemispherical photographs. Agr. Forest Meteorol. $148,644-655$

Edelsbrunner, H., Mücke, E.P., 1994. Three-dimensional alpha shapes. ACM Trans. Graph. (TOG) 13, 43-72

Gonsamo, A., Pellikka, P., 2009. The computation of foliage clumping index using hemispherical photography. Agr. Forest Meteorol. 149, 1781-1787.

Grau, E., Durrieu, S., Fournier, R., Gastellu-Etchegorry, J.P., Yin, T.G., 2017. Estimation of $3 \mathrm{D}$ vegetation density with Terrestrial Laser Scanning data using voxels. A sensitivity analysis of influencing parameters. Remote Sens. Environ. 191, 373-388.

Hosoi, F., Omasa, K., 2006. Voxel-based 3-D modeling of individual trees for estimating leaf area density using high-resolution portable scanning lidar. IEEE Trans. Geosci. Remote 44, 3610-3618.

Hu, R., Luo, J., Yan, G., Zou, J., Mu, X., 2016. Indirect measurement of forest leaf area index using path length distribution model and multispectral canopy imager. IEEE J. Sel Top Appl. 9, 2532-2539.

Hu, R., Yan, G., Mu, X., Luo, J., 2014. Indirect measurement of leaf area index on the basis of path length distribution. Remote Sens. Environ. 155, 239-247.

Hu, R., Yan, G., Nerry, F., Liu, Y., Jiang, Y., Wang, S., Chen, Y., Mu, X., Zhang, W., Xie, D., 2018. Using airborne laser scanner and path length distribution model to quantify clumping effect and estimate leaf area index. IEEE Trans. Geosci. Remote 56, 3196-3209.

Jonckheere, I., Fleck, S., Nackaerts, K., Muys, B., Coppin, P., Weiss, M., Baret, F., 2004. Review of methods for in situ leaf area index determination: Part I. Theories, sensors and hemispherical photography. Agr. Forest Meteorol. 121, 19-35.

Jupp, D.L.B., Culvenor, D.S., Lovell, J.L., Newnham, G.J., Strahler, A.H., Woodcock, C.E., 2009. Estimating forest LAI profiles and structural parameters using a ground-based laser called 'Echidna (R)'. Tree Physiol. 29, 171-181.

Koch, B., Heyder, U., Weinacker, H., 2006. Detection of individual tree crowns in airborne lidar data. Photogramm Eng. Rem Sens. 72, 357-363.
Leblanc, S.G., Chen, J.M., Fernandes, R., Deering, D.W., Conley, A., 2005. Methodology comparison for canopy structure parameters extraction from digital hemispherical photography in boreal forests. Agr. Forest Meteorol. 129, 187-207.

Leblanc, S.G., Fournier, R.A., 2014. Hemispherical photography simulations with an architectural model to assess retrieval of leaf area index. Agr. Forest Meteorol. 194, 64-76.

Lin, Y., West, G., 2016. Retrieval of effective leaf area index (LAIe) and leaf area density (LAD) profile at individual tree level using high density multi-return airborne LiDAR. Int. J. Appl. Earth Obs. 50, 150-158.

Macfarlane, C., Ryu, Y., Ogden, G.N., Sonnentag, O., 2014. Digital canopy photography: exposed and in the raw. Agr. Forest Meteorol. 197, 244-253.

Moorthy, I., Miller, J.R., Hu, B.X., Chen, J., Li, Q.M., 2008. Retrieving crown leaf area index from an individual tree using ground-based lidar data. Can. J. Remote Sens. 34 320-332.

Mu, X., Hu, R., Zeng, Y., McVicar, T.R., Ren, H., Song, W., Wang, Y., Casa, R., Qi, J., Xie, D., Yan, G., 2017. Estimating structural parameters of agricultural crops from ground-based multi-angular digital images with a fractional model of sun and shade components. Agr. Forest Meteorol. 246, 162-177.

Najjar, G., Colin, J., Kastendeuch, P., Ngao, J., Saudreau, M., Landes, T., Ameglio, T., Luhahe, R., Guillemin, S., Schreiner, G., 2015. A three years long fieldwork experiment to monitor the role of vegetation on the urban climate of the city of Strasbourg, France, International conférence on urban climate, 12th symposium, Toulouse, France 20-24 july.

Newnham, G., Armston, J., Muir, J., Goodwin, N., Tindall, D., Culvenor, D., Püschel, P., Nyström, M., Johansen, K., 2012. Evaluation of terrestrial laser scanners for measuring vegetation structure, CSIRO Sustainable Agriculture Flagship.

Nilson, T., 1999. Inversion of gap frequency data in forest stands. Agr. Forest Meteorol. 98-99, 437-448.

Nowak, D.J., Crane, D.E., Stevens, J.C., Hoehn, R.E., Walton, J.T., Bond, J., 2008. A ground-based method of assessing urban forest structure and ecosystem services.

Peper, P.J., McPherson, E.G., 1998. Comparison of five methods for estimating leaf area index of open-grown deciduous trees. J. Arboric. 24, 98-111.

Peper, P.J., McPherson, E.G., 2003. Evaluation of four methods for estimating leaf area of isolated trees. Urban For. Urban Gree 2, 19-29.

Pimont, F., Dupuy, J.L., Rigolot, E., Prat, V., Piboule, A., 2015. Estimating leaf bulk density distribution in a tree canopy using terrestrial LiDAR and a straightforward calibration procedure. Remote Sens. 7, 7995-8018.

Pisek, J., Ryu, Y., Alikas, K., 2011. Estimating leaf inclination and G-function from leveled digital camera photography in broadleaf canopies. Trees-Struct. Funct. 25, 919-924.

Ren, H., Yan, G., Liu, R., Nerry, F., Li, Z.-L., Hu, R., 2013. Impact of sensor footprint on measurement of directional brightness temperature of row crop canopies. Remote Sens. Environ. 134, 135-151.

Roberts, S.D., Dean, T.J., Evans, D.L., 2003. Family influences on leaf area estimates derived from crown and tree dimensions in Pinus taeda. Forest Ecol. Manage. 172, 261-270.

Roberts, S.D., Dean, T.J., Evans, D.L., McCombs, J.W., Harrington, R.L., Glass, P.A., 2005 Estimating individual tree leaf area in loblolly pine plantations using LiDAR-derived measurements of height and crown dimensions. Forest Ecol. Manage. 213, 54-70.

Ryu, Y., Sonnentag, O., Nilson, T., Vargas, R., Kobayashi, H., Wenk, R., Baldocchi, D.D., 2010. How to quantify tree leaf area index in an open savanna ecosystem: a multi-instrument and multi-model approach. Agric. Forest Meteorol. 150, 63-76.

Simioni, G., Gignoux, J., Le Roux, X., Appe, R., Benest, D., 2004. Spatial and temporal variations in leaf area index, specific leaf area and leaf nitrogen of two co-occurring savanna tree species. Tree Physiol. 24, 205-216.

Simpson, J.R., 1998. Urban forest impacts on regional cooling and heating energy use Sacramento County case study. J. Arboric. 24, 201-209.

Sonohat, G., Sinoquet, H., Kulandaivelu, V., Combes, D., Lescourret, F., 2006. Three-dimensional reconstruction of partially 3D-digitized peach tree canopies. Tree Physiol. 26, 337-351.

Stenberg, P., 1996. Correcting LAI-2000 estimates for the clumping of needles in shoots of conifers. Agr. Forest Meteorol. 79, 1-8.

Stenberg, P., 1996. Simulations of the effects of shoot structure and orientation on vertical gradients in intercepted light by conifer canopies. Tree Physiol. 16, 99-108.

Strahler, A.H., Jupp, D.L.B., Woodcock, C.E., Schaaf, C.B., Yao, T., Zhao, F., Yang, X.Y., Lovell, J., Culvenor, D., Newnham, G., Ni-Miester, W., Boykin-Morris, W., 2008. Retrieval of forest structural parameters using a ground-based lidar instrument (Echidna (R)). Can. J. Remote Sens. 34, S426-S440.

Tian, Y., Zheng, Y., Zheng, C.M., Xiao, H.L., Fan, W.J., Zou, S.B., Wu, B., Yao, Y.Y., Zhang, A.J., Liu, J., 2015. Exploring scale-dependent ecohydrological responses in a large endorheic river basin through integrated surface water-groundwater modeling. Water Resour. Res. 51, 4065-4085.

Wang, C., Menenti, M., Stoll, M.P., Feola, A., Belluco, E., Marani, M., 2009. Separation of ground and low vegetation signatures in LiDAR measurements of salt-marsh environments. IEEE Trans. Geosci. Remote 47, 2014-2023.

Weiss, M., Baret, F., Smith, G.J., Jonckheere, I., Coppin, P., 2004. Review of methods for in situ leaf area index (LAI) determination: Part II. Estimation of LAI, errors and sampling. Agr. Forest Meteorol. 121, 37-53.

Xie, D.H., Wang, Y., Hu, R.H., Chen, Y.M., Yan, G.J., Zhang, W.M., Wang, P.J., 2017. Modified gap fraction model of individual trees for estimating leaf area using terrestrial laser scanner. J. Appl. Remote Sens. 11.

Yan, G., Hu, R., Wang, Y., Ren, H., Song, W., Qi, J., Chen, L., 2016. Scale effect in indirect measurement of leaf area index. IEEE Trans. Geosci. Remote 54, 3475-3484

Yao, T., Yang, X.Y., Zhao, F., Wang, Z.S., Zhang, Q.L., Jupp, D., Lovell, J., Culvenor, D., Newnham, G., Ni-Meister, W., Schaaf, C., Woodcock, C., Wang, J.D., Li, X.W., 
Strahler, A., 2011. Measuring forest structure and biomass in New England forest stands using Echidna ground-based lidar. Remote Sens. Environ. 115, 2965-2974.

Zeng, Y., Li, J., Liu, Q., Hu, R., Mu, X., Fan, W., Xu, B., Yin, G., Wu, S., 2015. Extracting leaf area index by sunlit foliage component from downward-looking digital photography under clear-sky conditions. Remote Sens. 7, 13410. 